\title{
Sorafenib inhibits tumor growth and vascularization of rhabdomyosarcoma cells by blocking IGF-IR-mediated signaling
}

\author{
Wessen Maruwge' \\ Pádraig D'Arcy' \\ Annika Folin ${ }^{1,2}$ \\ Slavica Brnjic' \\ Johan Wejde' \\ Anthony Davis' \\ Fredrik Erlandsson ${ }^{3}$ \\ Jonas Bergh ${ }^{1,2}$ \\ Bertha Brodin' \\ 'Department of Oncology \\ and Pathology, Karolinska \\ Institutet, Stockholm, Sweden; \\ ${ }^{2}$ Radiumhemmet, Karolinska \\ University Hospital, Stockholm, \\ Sweden; ${ }^{3}$ Bayer Pharmaceutical \\ Corporation, Sweden
}

\begin{abstract}
The growth of many soft tissue sarcomas is dependent on aberrant growth factor signaling, which promotes their proliferation and motility. With this in mind, we evaluated the effect of sorafenib, a receptor tyrosine kinase inhibitor, on cell growth and apoptosis in sarcoma cell lines of various histological subtypes. We found that sorafenib effectively inhibited cell proliferation in rhabdomyosarcoma, synovial sarcoma and Ewing's sarcoma with $\mathrm{IC}_{50}$ values $<5 \mu \mathrm{M}$. Sorafenib effectively induced growth arrest in rhabdomyosarcoma cells, which was concurrent with inhibition of Akt and Erk signaling. Studies of ligand-induced phosphorylation of Erk and Akt in rhabdomyosarcoma cells showed that insulin-like growth factor-1 is a potent activator, which can be blocked by treatment with sorafenib. In vivo sorafenib treatment of rhabdomyosarcoma xenografts had a significant inhibitory effect on tumor growth, which was associated with inhibited vascularization and enhanced necrosis in the adjacent tumor stroma. Our results demonstrate that in vitro and in vivo growth of rhabdomyosarcoma can be suppressed by treatment with sorafenib, and suggests the possibilities of using sorafenib as a potential adjuvant therapy for the treatment of rhabdomyosarcoma.
\end{abstract}

Keywords: soft tissue sarcoma, kinase inhibitors, targeted therapy, vascularization

\section{Introduction}

The recent identification of broad range multikinase inhibitors has raised the possibility for potential targeted therapies against tumors which display dependency on growth factor-mediated signaling. Many cancer cells utilize numerous receptor tyrosine kinases such as platelet-derived growth factor receptor- $\beta$ (PDGFR $\beta)$, c-KIT and vascular endothelial growth factor receptor (VEGFR), to transmit growth signals from the cell surface to the intra cellular milieu to mediate numerous biological responses (Amit et al 2007; Do et al 2007). Two of the most characterized receptor tyrosine kinase-mediated signaling cascades are the Ras/Raf/MEK/Erk and Ras/PI3K/Akt pathways which are involved in promoting cell proliferation (Chambard et al 2007) and cell survival (Downward 1998; Xue et al 2000) respectively. Receptor tyrosine kinases induced activation of the Ras/Raf/MEK/Erk pathway promotes cell proliferation by inducing the activating phosphorylation and nuclear localization of Erk, which promotes cell cycle progression by mediating transcriptional activation of the cyclin D1 gene and by promoting the disassociation of E2F1 from the negative regulation of $\mathrm{Rb}$ (Chambard et al 2007). In contrast, the activation of Akt promotes cell survival as opposed to proliferation. Phosphorylated Akt promotes stabilization of HDM2, the negative regulator of $\mathrm{p} 53$, promotes sequesterization of the pro apoptotic Bad protein and promotes enhanced protein translation via the mTOR pathway (Datta et al 1997; del Peso et al 1997; Ashcroft et al 2002; Faivre et al 2006). Crosstalk also exists between the Erk and Akt pathways via MEK (Misra and Pizzo 2004; Myhre et al 2004; Merighi et al 2006), 
thus providing further levels of regulation. In light of their importance in cancer, both pathways serve as useful molecular targets for the development of targeted therapies.

BAY 43-9006/Sorafenib/Nexavar ${ }^{\circledR}$ (hereafter referred to as sorafenib), is a small molecule, multi kinase inhibitor. Although initially identified as a Raf inhibitor, subsequent molecular studies have shown that it is also a potent inhibitor of several receptor tyrosine kinases involved in tumor progression, including VEGFR-2 and -3, PDGFR $\beta$, c-KIT and FLT-3 (Lowinger et al 2002; Wilhelm et al 2004, 2006). Sorafenib was shown to effectively block the Raf/MEK/Erk signaling pathway and to have broad anti-tumor activity in preclinical studies (Karasarides et al 2004; Wilhelm et al 2004; Panka et al 2006).

The efficacy of sorafenib in clinical trials for the treatment of solid tumors has been most favorable for renal cell carcinoma (Ratain et al 2006; Bracarda et al 2007; Escudier et al 2007; Llovet et al 2007). In a phase III trial, including patients with advance clear-cell renal-cell carcinoma, treatment with sorafenib alone as second-line therapy prolonged the progression-free survival, resulting in 74\% (sorafenib) and 53\% (placebo) stable disease, and a disease control rate of $62 \%$ (sorafenib) and 37\% (placebo) (Escudier et al 2007). Another phase III clinical trial, including patients with advanced hepatocellular carcinoma with no previous systemic treatment, showed prolonged survival in patients receiving treatment with sorafenib versus placebo, resulting in a $44 \%$ increase in overall survival (Llovet et al 2007).

Clinical trials evaluating the activity of sorafenib in soft tissue sarcomas have been conducted. A phase II trial on advanced gastrointestinal stromal tumors (GIST) that express c-Kit and with resistance to imatinib and sunitinib, resulted in $14 \%$ partial response, $62 \%$ stable disease, $24 \%$ progressive disease, and a disease control rate of $76 \%$ (Wiebe et al 2008). On non-GIST sarcomas, a phase II trial showed a 15\% and 5\% response in angiosarcomas and leiomyosarcomas, respectively (D'Adamo et al 2007). It was recently reported that malignant peripheral nerve sheath tumor (MPNST) cell lines are sensitive to sorafenib, whereas liposarcoma cell lines are resistant to treatment with sorafenib (Ambrosini et al 2008). However, a phase II study of sorafenib in patients with leiomyosarcoma, malignant fibrous histiocytoma (MFH), MPNST, angiosarcoma and synovial sarcoma, resulted in a $14 \%$ and $6 \%$ response rate in patients with angiosarcoma and leiomyosarcoma, respectively, but only minor responses were observed in patients with MPNST and synovial sarcoma (Maki et al 2008). Another phase II study, in patients with advanced soft tissue sarcomas, did not result in RECIST response. However, a notable progression free survival was observed in patients with angiosarcoma and hemangiopericytoma (Ryan et al 2008).

In this study we investigate and evaluate the effect of sorafenib in 13 soft tissue sarcoma cell lines of four different histological subtypes in vitro and in tumor xenografts.

\section{Materials and methods Cell lines}

Panels of 13 sarcoma cell lines (three rhabdomyosarcomas, two synovial sarcomas, four Ewing's sarcomas, and four osteosarcomas) were analyzed. Rhabdomyosarcoma cell lines RH30, RD, and RMS were obtained from Molecular Cytogenetics Unit at Institute of Cancer Research (Sutton, UK). The synovial sarcoma cell line CME-1 was obtained from the Instituto Nazionale per lo Studio e la Cura dei Tumori (Milan, Italy). Ewing's sarcoma cell lines SK-ES-1, SK-N-MC, RD-ES and Lap 35, and osteosarcoma cell lines IOR-OS9 and IOR-OS10 were obtained from the Laboratory of Oncologic Research, Orthopaedic, Rizzoli Institute (Bologna, Italy). Osteosarcoma cell lines SAOS2 and U2OS were obtained from ATCC culture collection. All cell lines grow as adherent cultures and were passaged in optimized mediums supplemented with 100 units/ml penicillin, $100 \mu \mathrm{g} / \mathrm{ml}$ streptomycin (Sigma-Aldrich, St. Louis, MO, USA) and 10\%-20\% heat inactivated fetal bovine serum (FBS) (High clone III, HyClone, Cramlington, UK). Information about the cell lines, their genetic and phenotypic characteristics is provided in Table 1.

\section{Drugs}

Sorafenib was kindly provided by Bayer Pharmaceuticals Corporation (West Haven, CT, USA). For in vitro assays, a $10 \mathrm{mM}$ stock was prepared in dimethyl-sulfoxide (DMSO) (Sigma-Aldrich), newly prepared before the initiation of each assay, from which working solutions were carefully set immediately before use according to desired concentrations. Sorafenib is light sensitive in solution and was therefore kept in dark during the course of the experiments. For in vivo studies, sorafenib was newly prepared in a vehicle composed of Cremophor EL/ethanol/water (12.5\%-12.5\%-75\%) for daily per oral administration.

\section{In vitro cytotoxicity assays}

Cells were seeded in 96 well plates and incubated overnight to become adherent before treatment with sorafenib. Sorafenib was added to final concentrations of $0-5 \mu \mathrm{M}$. Cell viability and proliferation was determined after $48 \mathrm{hrs}$ by the capability of viable cells to cleave tetrazolium salt into formazan 


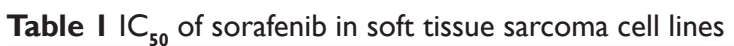

\begin{tabular}{llll}
\hline & Cell line & Genetics & IC $_{50}(\mu$ M Sorafenib) \\
\hline Rhabdomyosarcoma & RMS (a) & PAX3/7-FKHR & 3.9 \\
Rhabdomyosarcoma & RH30 (a) & PAX3/7-FKHR & 5 \\
Rhabdomyosarcoma & RD (e) & $22 q-$ & 4.5 \\
Synovial sarcoma & SYO-I & SSI8/SSX2 & 2.4 \\
Synovial sarcoma & CME-I & SSI8/SSX2 & 2.8 \\
Ewing's sarcoma & SK-N-MC & EWS-FLI-I (tI) & 2.4 \\
Ewing's sarcoma & SK-ES-I & EWS-FLI-I (t2) & $>5$ \\
Ewing's sarcoma & RD-ES & EWS-FLI-I (t2) & 3 \\
Ewing's sarcoma & LAP-35 & EWS-FLI-I (t2) & $>5$ \\
Osteosarcoma & SAOS2 & complex & $>5$ \\
Osteosarcoma & U2OS & complex & $>5$ \\
Osteosarcoma & IOR-OS9 & complex & $>5$ \\
Osteosarcoma & IOR-OSI0 & complex & $>5$
\end{tabular}

(WST-1 Assay, Roche, Basel, Switzerland), according to manufacturer's instructions. Percentage of viability after drug administration was calculated in relation to untreated control (100\% viability). Each assay was repeated a minimum of four times to confirm trends and the average concentration at which $50 \%$ of the cells are inhibited $\left(\mathrm{IC}_{50}\right)$ determined.

\section{Immunofluorescence}

Cells were plated in eight well chamber slides (Falcon, BD Biosciences, San Jose, CA, USA) and allowed to adhere over night. Cells were fixed in ice cold acetone/methanol (1:1) for 15 minutes at $-20{ }^{\circ} \mathrm{C}$, permebealized in $\mathrm{PBS} / 0.25 \%$ Triton-X 100 and blocked in blocking buffer (2\% BSA, 5\% glycerol, $0.2 \%$ Tween 20 and $0,02 \%$ Na-azide). Cells were incubated with primary antibody overnight at $4{ }^{\circ} \mathrm{C}$, washed with PBS $/ 0.05 \%$ Tween 20 , incubated with secondary antibody for 45 minutes (anti-rabbit DyLight Alexa 488 FITC; Pierce Biotechnology Inc., Rockford, IL, USA or anti-mouse Texas Red; Vector laboratories, Burlingame, CA, USA), washed and mounted with Vectashield mounting media containing DAPI (Vector Laboratories). Cells were stained with the following antibodies: IGF1R $\beta$ (1:20, Cell Signaling Technology Inc., Danvers, MA, USA), VEGFR2 (1:50, Cell Signaling Technology Inc.), PDGFR $\beta$ (1:50, Sigma-Aldrich), FLT-3 (1:50, Cell Signaling Technology Inc.,) and c-KIT (1:50, BiosourceInvitrogen, Carlsbad, CA, USA).

\section{FACS analysis}

Cells were seeded and incubated over night to become adherent before treatment with sorafenib. Sorafenib was added to final concentrations of $0-5 \mu \mathrm{M}$ for $48 \mathrm{hrs}$.
To estimate DNA fragmentation by apoptosis, cells were stained with propidium iodide (PI) and floating and attached cells were collected by trypsinization and fixed in $70 \%$ ice cold ethanol for $24 \mathrm{hrs}$. Cells were stained with $50 \mu \mathrm{g} / \mathrm{ml}$ PI supplemented with recombinant $100 \mu \mathrm{g} / \mathrm{ml}$ RNAse for 45 minutes at room temperature and DNA fragmentation by apoptosis was analyzed as the sub-G1 cell fraction.

For Annexin V staining, cells were harvested by trypsinisation and stained with Annexin $\mathrm{V}$ apoptosis detection kit (Sigma-Aldrich) according to manufacturer's protocol, and analyzed by FACS, for detection of cells during early phases of apoptosis.

\section{Western blot and phosphorylation analysis}

Cells were seeded and incubated over night to become adherent, followed by starvation in serum deficient medium for $24 \mathrm{hrs}$ prior to substitution with FBS-enriched medium. Sorafenib was added to final concentrations of $0-5 \mu \mathrm{M} 30$ minutes before serum stimulation. Cells were then harvested in $2 \mathrm{x}$ loading buffer, containing $10 \% \beta$-mercaptoethanol, sonicated, heated for 10 minutes at $90{ }^{\circ} \mathrm{C}$ and the cell lysates were separated by SDS-PAGE (Invitrogen) on 4\%-12\% gradient gels under denaturing conditions and transferred onto PVDF membranes (Amersham, Buckinghamshire, UK). After blocking in 5\% BSA (Sigma-Aldrich), the PVDF filters were incubated over night at $4{ }^{\circ} \mathrm{C}$ with the primary antibody anti-phospo-Erk or anti-phospho-Akt (Cell Signaling Technology Inc.), total AKT or total ERK (Sigma-Aldrich). After a series of washes in PBS $/ 0.1 \%$ Tween 20 (PBS-T), the membranes were incubated $1 \mathrm{hr}$ with secondary antibody conjugated to horseradish 
peroxidase (Pierce Biotechnology Inc., Rockford, IL, USA). Filters were subsequently washed in PBS-T and developed using chemiluminescence western blotting detection reagents (Pierce Biotechnology Inc.,).

\section{Activation of MAPK and AKT by ligand stimulation}

Cells were seeded and incubated over night to become adherent, followed by starvation in serum deficient medium for $24 \mathrm{hrs}$ prior to treatment with $0-5 \mu \mathrm{M}$ sorafenib for $4 \mathrm{hrs}$, followed by ligand treatment $(50 \mathrm{ng} / \mathrm{ml})$ for 30 minutes. The cells were then harvested in $2 \mathrm{x}$ loading buffer and the lysates were separated by SDS-PAGE, as described above. Cells were stimulated with FLT-3, SCF, VEGF, PDGF (Immuno Tools, Friesoythe, Germany), and insulin-like growth factor (IGF) (Sigma-Aldrich).

\section{Xenografts and sorafenib treatment}

The described animal studies were approved by the ethics committee Stockholms Norra Djurförsöksetiska Nämnd (N113/04).

BALB/c SCID mice ( $~ 8$ weeks old, $20-30 \mathrm{~g}$ ) were obtained from and the animal experiments were carried out at the Microbiology and Tumor biology Center (MTC) Animal Facility, Karolinska Institutet, Sweden. The animals were cared for by trained animal keepers and the health status of the animals was supervised carefully.

Each mouse (6 per group) was inoculated subcutaneously with $5 \times 10^{6}$ cells resuspended in $100 \mu 1$ matrigel (BD Biosciences, San Jose, CA, USA) in the right flank. Treatment with sorafenib was initiated when tumors were palpable (day 8 after inoculation) by daily per oral administration of sorafenib (40, 60, $80 \mathrm{mg} / \mathrm{kg}$ body weight) or placebo (vehicle only) for a total of 7 days. Tumor dimensions were recorded twice weekly. At the end point of the experiment, mice were euthanized by cervical dislocation and each tumor was excised and fixed in formalin. Tumor volumes were calculated using the equation $\left(l \times w^{2}\right) / 2$, where $l$ and $w$ represent the largest and smallest dimensions at each measurement. The effect of drug treatment on tumor growth was analyzed statistically with individual group comparison and evaluated with independent samples T-test by SPSS 15.0 (SPSS Inc., Chicago, IL, USA).

\section{Preparation of paraffin tumor tissue sections}

Animals were euthanized by cervical dislocation and the tumor tissue was excised and fixed in formalin and embedded in paraffin. The tumors were cut in $4 \mu \mathrm{m}$ sections and the sections were placed on object slides. Paraffin was cleared with xylene and the tumor sections were re-hydrated and stained with Mayers hematoxylin and eosin.

\section{Immunohistochemistry of paraffin tumor tissue sections}

Sections were studied immunohistochemically, using the AvidinBiotin Complex method (VECTASTAIN Elite ABC Kit; Vector Laboratories). Heat-induced epitope retrieval with citrate buffer ( $\mathrm{pH} 6)$ was performed for these sections via microwave oven at $98{ }^{\circ} \mathrm{C}$ for 20 minutes, or pronase for 15 minutes. Endogenous peroxidase activity was blocked with $\mathrm{H}_{2} \mathrm{O}_{2}$. Peroxidase activity was developed with 3-3-diaminobenzidine (Sigma-Aldrich) to obtain a brown end product. Representative sections were stained with the following antibodies: Ki-67 (MIB-1, 1:500, DAKO, Glostrup, Denmark) and PECAM-1 (M-20, 1:50, Santa Cruz Biotechnology Inc., Santa Cruz, CA, USA).

\section{Results}

\section{Sorafenib inhibits cell proliferation}

\section{in synovial sarcoma, rhabdomyosarcoma} and Ewing's sarcoma cell lines at clinically relevant doses

Rhabdomyosarcomas, synovial sarcomas, osteosarcomas and Ewing's sarcomas typically express multiple receptor tyrosine kinases all of which are potential targets for inhibition by sorafenib (Ricotti et al 1998; Handa et al 2000; Landuzzi et al 2000; Zhang et al 2000; Gordon et al 2001; Lowinger et al 2002; Kawai et al 2004; Tamborini et al 2004; Gee et al 2005; Kreuter et al 2006; Armistead et al 2007; Do et al 2007). With this in mind, we evaluated the anti-proliferative effect of sorafenib on a panel of soft tissue tumor cell lines. Cells were exposed to increasing concentrations of sorafenib for $48 \mathrm{hrs}$ with values greater than $5 \mu \mathrm{M}$ considered beyond the scope of clinical relevance. The ability of sorafenib to inhibit cell proliferation was determined by WST-1 assay, a colorimetric assay that measures the metabolic activity which correlates to cell viability. We found that sorafenib inhibited cell growth in a dose dependent manner in all rhabdomyosarcoma (3 of 3); in all synovial sarcoma ( 2 of 2 ), and in $50 \%$ of Ewing's sarcoma cell lines ( 2 of 4 ), with $\mathrm{IC}_{50}$ values below $5 \mu \mathrm{M}$ (Table 1 ). All four osteosarcoma cell lines were refractive to sorafenib treatment within these concentration ranges (Table 1). Since all three rhabdomyosarcoma cells responded we chose these 
cell lines for further investigations. Microscopic analysis of cells showed a dose dependent decrease in cell numbers in sorafenib-treated cells (Figure 1A). We evaluated the induction of apoptosis following treatment with sorafenib in the rhabdomyosarcoma cell line RMS by annexin V staining, as an indicator of early apoptosis and propidium iodide staining for DNA fragmentation. Treatment with sorafenib for $48 \mathrm{hrs}$ resulted only in a moderate increase in both annexin $\mathrm{V}$ staining and DNA cleavage when compared to mock-treated control cells (Figures 1B-C). Taken together our data demonstrates that sorafenib primarily induces growth arrest as opposed to apoptosis in rhabdomyosarcoma cells.

\section{Sorafenib inhibits Erk and Akt activation in rhabdomyosarcoma cells}

In order to gain further insight into the downstream effects of sorafenib in rhabdomyosarcoma, we analyzed the activation of Erk and Akt in response to serum stimulation

A

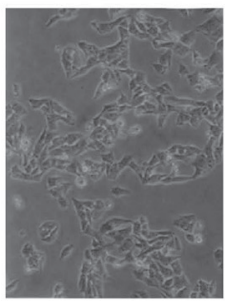

Sorafenib $(\mu \mathrm{M})$ :

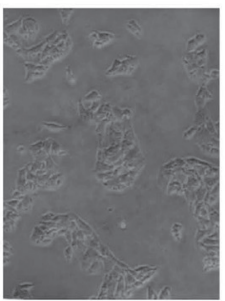

1

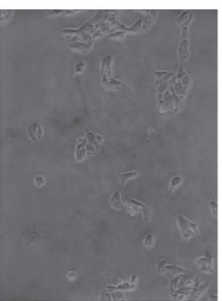

3

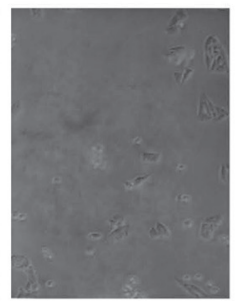

5

B

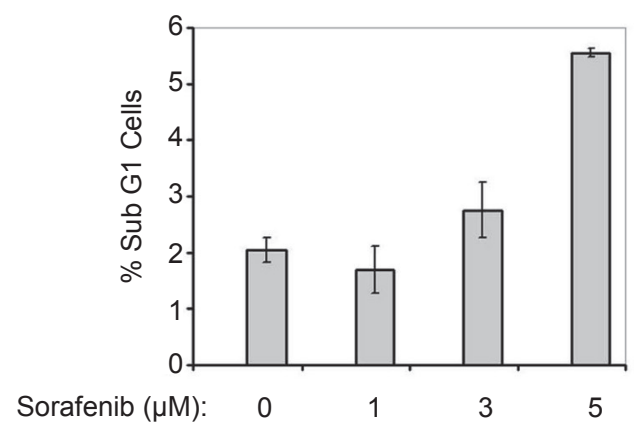

C

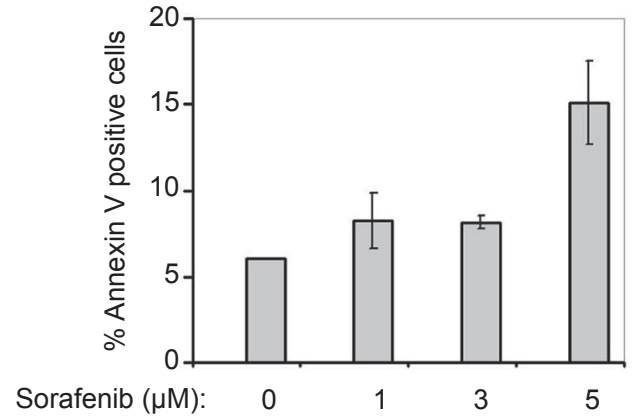

Figure I Effect of sorafenib on cell cycle in alveolar rhabdomyosarcoma (RMS) cells. A) Sorafenib inhibits proliferation of RMS in a dose dependent manner. Rhabdomyosarcoma cells were cultured and treated with $0-5 \mu \mathrm{M}$ sorafenib for $48 \mathrm{hrs}$ and visualized using light microscopy. B) Estimation of sub-GI content. Cells were treated as above and sub-GI fraction of cells was determined by PI staining and FACS analysis. C) Annexin V staining of sorafenib-treated RMS cells. Cells were treated with sorafenib as above and the level of Annexin $V$ staining was determined by FACS analysis.

Abbreviation: Pl, propidium iodide. 
in the absence and presence of sorafenib. Western blot analysis of Erk and Akt phosphorylation showed that sorafenib effectively inhibited serum-induced phosphorylation in a dose dependent manner (Figure 2A). Sorafenib totally inhibited Erk and Akt phosphorylation at $3 \mu \mathrm{M}$ without affecting the total levels. We investigated the effect of treatment with sorafenib on the time dependent inhibition of Erk and Akt activation following serum stimulation. Serum-induced phosphorylation of Erk reached maximum phosphorylation $10 \mathrm{~min}$ post-serum addition with levels rapidly decreasing thereafter, whereas Akt phosphorylation displayed a slower induction profile with levels rising over the period of the time course (Figure 2B). Pretreatment with sorafenib effectively blocked both Erk and Akt phosphorylation following serum stimulation. Taken together our results demonstrate that sorafenib effectively blocks serum-induced Erk and Akt activation in rhabdomyosarcoma cells.

A

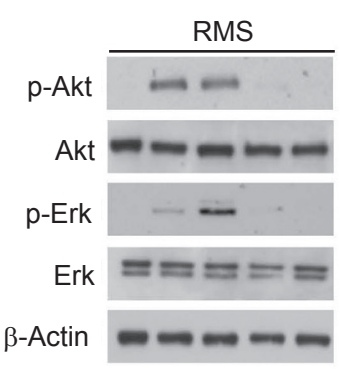

Serum: -+++

Sorafenib $(\mu \mathrm{M}):-\begin{array}{lllll}0 & 1 & 3 & 5\end{array}$

B

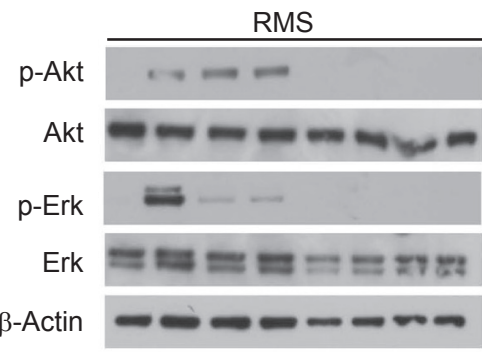

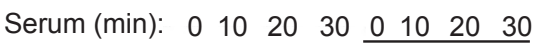

+Sorafenib

Figure 2 Sorafenib inhibits Erk and Akt activation in alveolar rhabdomyosarcoma (RMS) cells. A) RMS cells were starved for $24 \mathrm{hrs,} \mathrm{treated} \mathrm{with} \mathrm{0-5} \mu \mathrm{M}$ sorafenib for $4 \mathrm{hrs}$ and simulated with serum for 10 minutes. Cells were harvested for western blotting by direct lysis in loading buffer. Levels of phosphorylated Erk and Akt were detected using phosphor specific antibodies. Levels of total Erk and Akt were used as a loading control. B) RMS cells were serum starved for $24 \mathrm{hrs}$, pre-treated with $5 \mu \mathrm{M}$ sorafenib and stimulated with serum for the indicated time points. Levels of phosphor Erk and Akt were detected as above.

\section{Sorafenib inhibits IGF-I-induced Erk and Akt signaling in rhabdomyosarcoma cell lines}

Sorafenib has been shown to inhibit Erk and Akt phosphorylation in response to activation of tyrosine kinase receptors (Wilhelm et al 2004; Sridhar et al 2005; Adnane et al 2006; Flaherty 2006; Tong et al 2006; Yu et al 2006; Zhong and Bowen 2007; Ammoun et al 2008). Analysis of receptor tyrosine expression showed the presence of high levels of c-KIT, PDGFR $\beta$, FLT-3 and VEGFR2 in all rhabdomyosarcoma cell lines (Figure 3, Table 2). We also included IGF-1R since over expression has been previously observed in rhabdomyosarcoma (El-Badry et al 1990; Minniti et al 1992, 1994; Shapiro et al 1994). High levels of IGF-1R expression was observed in all rhabdomyosarcoma cell lines as indicated by high levels of cell surface staining. Next we evaluated Erk and Akt phosphorylation in response to ligand addition. Surprisingly, ligand stimulation induced by SCF, VEGF, PDGF and FLT-3 only induced weak phosphorylation of Erk and Akt in all three rhabdomyosarcoma cell lines when compared with growth serum. Since our previous data showed that IGF-1R is expressed at high levels in rhabdomyosarcoma cells, we analyzed the effect of IGF-1 stimulation on Erk and Akt phosphorylation in the presence and absence of sorafenib. In contrast to the previous ligands, IGF-1 was a potent inducer of Erk and Akt phosphorylation in all cell lines with IGF-1 inducing Erk and Akt phosphorylation to levels similar to that observed in serum stimulated cells. Treatment with $5 \mu \mathrm{M}$ sorafenib effectively blocked IGF-1-mediated activation of Akt and Erk in RMS cells (Figure 4A). Sorafenib also blocked IGF-1-mediated activation of Erk in RH30 and RD cells (Figures 4B and C), however, Akt phosphorylation was still observed (Figures 4B and C). Our results show that IGF-1 is a potent activator of Erk and Akt in rhabdomyosarcoma RMS cells which can be effectively blocked by sorafenib.

\section{Sorafenib inhibits IGF-I-induced cell growth in rhabdomyosarcoma cell lines}

To determine if sorafenib inhibited growth induced by IGF-1 stimulation, we preformed WST-1 cell proliferation assays on rhabdomyosarcoma cells which were grown in the presence and absence of sorafenib and with or without serum or IGF-1. Cells were starved and then allowed to grow in the presence or absence of serum or IGF-1, followed by treatment with $5 \mu \mathrm{M}$ sorafenib over a $48 \mathrm{hrs}$ period. Inhibition of cell proliferation by sorafenib was normalized to each of 


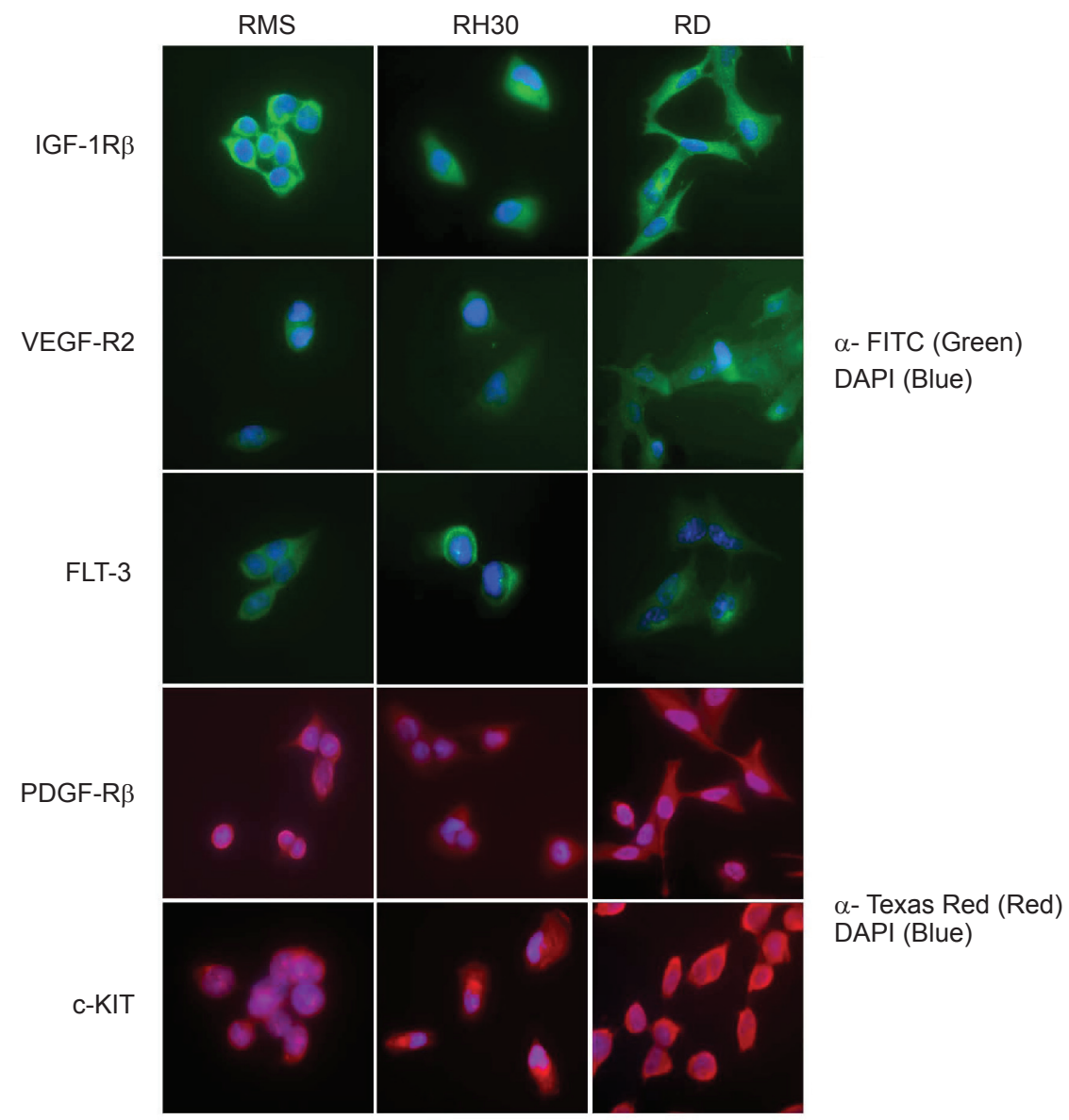

Figure 3 Expression of receptor tyrosine kinases in rhabdomyosarcoma cells. Rhabdomyosarcoma cells (RMS, RH30, and RD) were cultured in chamber slides, fixed and incubated with antibodies against the indicated receptor tyrosine kinase. Receptor tyrosine expression was visualized using the appropriate fluorescent conjugated secondary antibody.

the growth conditions. We found that sorafenib inhibited cell growth in normal conditions (as mentioned above) and to a greater extent in starved conditions and in cells grown in the presence of IGF-1 (Figure 5). Our data shows for the first time that sorafenib can inhibit cell proliferation induced by IGF-1 in rhabdomyosarcoma cells.

\section{Sorafenib inhibits tumor growth and vascularization in rhabdomyosarcoma xenografts}

To evaluate the antiproliferative effect of sorafenib in vivo, rhabdomyosarcoma xenografts were established in SCID mice, as described in materials and methods. Analysis of RMS xenograft tumors in placebo-treated mice showed the presence of large tumors, which appeared highly vascularized. There was a significant difference in tumor volume (at least four times as large) in sorafenib-treated mice when compared with placebo (Figure 6A). Upon macroscopic examination of the excised tumors, sorafenib-treated tumors were typically smaller and poorly developed, which was reflected in the administered dose. Hematoxilin and eosin staining of tumor tissue showed that histology of the untreated tumors (Figure 6B, upper left panel) had a classical alveolar structure

Table 2 Expression of RTKs in soft tissue sarcoma cell lines

\begin{tabular}{llllll}
\hline Cell line & IGF-IR & VEGF-R2 & FLT-3 & PDGF-R $\beta$ & c-KIT \\
\hline RMS (a) & + & + & + & + & + \\
RH30 (a) & + & + & + & + & + \\
RD (e) & + & + & + & + & + \\
SYO-I & + & + & + & + & + \\
CME-I & - & nd & nd & + & + \\
SK-N-MC & + & nd & nd & - & + \\
SK-ES-I & + & + & + & $+/-$ & + \\
RD-ES & + & nd & nd & - & + \\
LAP-35 & + & nd & nd & + & $+/-$ \\
\hline
\end{tabular}

+ : receptor tyrosine kinase is expressed.

-: receptor tyrosine kinase is not expressed.

nd: not determined. 
A

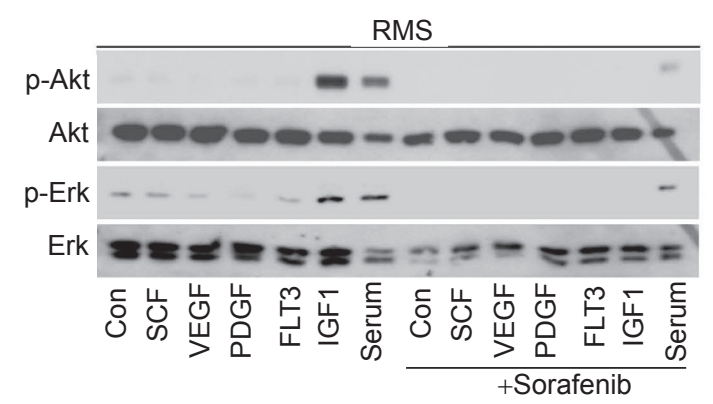

B

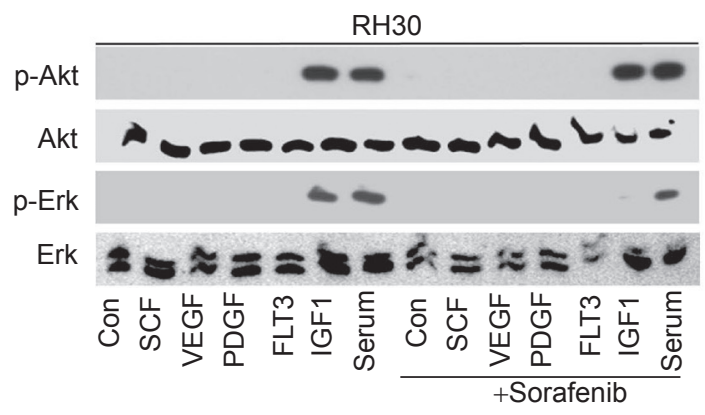

C

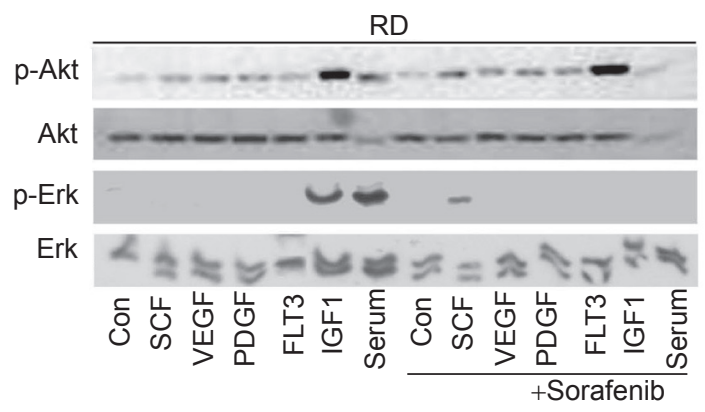

Figure 4 Sorafenib inhibits activation of Erk and Akt through IGF-I signaling in rhabdomyosarcoma cells. Rhabdomyosarcoma cells were starved for 24 hrs, treated with $5 \mu \mathrm{M}$ sorafenib and stimulated with $50 \mathrm{ng} / \mathrm{ml}$ of the appropriate ligand (SCF, VEGF, PDGF, FLT-3 or IGF-I) or serum for 30 minutes. Cells were lysed directly in loading buffer and immunoblotting of Erk and Akt phosphorylation was performed for A) RMS, B) RH30, and C) RD cells using phosphor-specific Erk and Akt antibodies. Levels of total Erk and Akt were used as loading controls.

Abbreviations: IGF, insulin-like growth factor; SCF, stem cell factor; VEGF, vascular endothelial growth factor; PDGF, platelet-derived growth factor; FLT3, FMS-like tyrosine kinase 3.

typical of RMS rhabdomyosarcoma, consisting of clusters of tumor cells surrounded by collagenous fibrovascular tissue (septa, S). Sorafenib-treated tumors (Figure 6B, upper right panel) displayed high levels of necrotic areas (N) around the septa, and were characterized by the presence of necrotic cells (picnotic, dark purple).

To assess cell proliferation in these tumors, paraffin embedded sections were stained immunohistochemically with the proliferation marker Ki-67. Placebo-treated tumors had high rates of cell proliferation as determined by the relatively high intensity of Ki-67 staining (Figure 6B, lower left panel, brown color). Tumors from sorafenib-treated

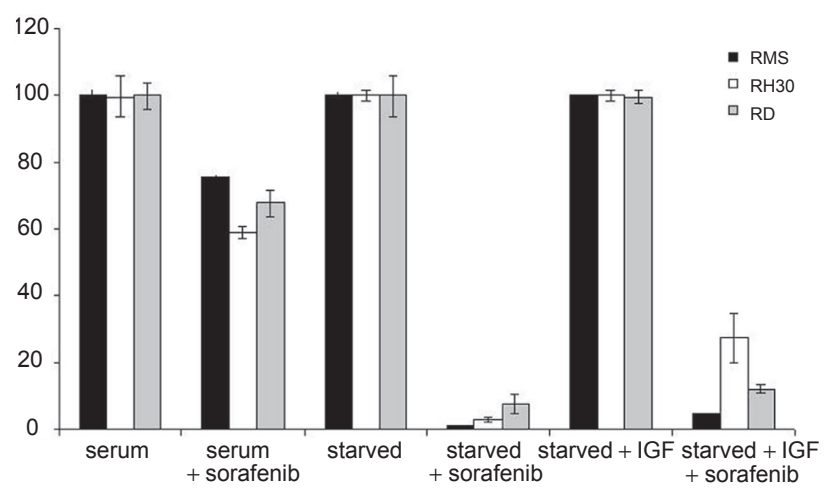

Figure 5 Sorafenib inhibits IGF- I-induced cell growth in rhabdomyosarcoma cell lines. Rhabdomyosarcoma cells (RMS, RH30, and RD) were grown in the presence or absence of serum or IGF, followed by treatment with $5 \mu \mathrm{M}$ sorafenib for $48 \mathrm{hrs}$. Cell proliferation was estimated using WST-I proliferation reagent as previously described. Abbreviation: IGF, insulin-like growth factor.

mice displayed striking lower levels of Ki-67 (Figure 4B, lower right panel). Furthermore, sorafenib-treated tumors showed areas of nonproliferative cells interspersed with proliferating cells.

We also evaluated the anti-angiogenic properties of sorafenib. Analysis of tumor vascularization was performed by staining of the mouse endothelial cells with an anti-CD31-antibody. The staining showed that vascularization in control tumors was characterized by the specific CD31-peroxidase staining of endothelial cells (E) lining the inner wall of blood vessels and interspaced in the septa (Figure 6C, left panel). These cells were less abundant or absent in blood vessels of tumors treated with sorafenib (Figure 6C, right panel).

\section{Discussion}

Sorafenib is a small multi-target inhibitor that has broad spectrum activity against several tyrosine kinases. Although initially described as a b-Raf inhibitor, sorafenib inhibits the activity of several cell surface receptor tyrosine kinases, such as VEGFR-2, FLT-3, c-KIT and PDGFR. A constitutive activation of receptor tyrosine kinases is a common feature of many types of cancers, particularly those of soft tissue origin where their concerted expression has been shown to promote tumor growth and survival (Zhang et al 2000; Lowinger et al 2002; Kawai et al 2004; Tamborini et al 2004; Gee et al 2005; Armistead et al 2007). As such small molecules which can inhibit one or all of these receptor tyrosine kinases may provide a means of inhibiting tumor cells by removing positive growth and survival signaling. With this in mind, we hypothesized that sorafenib would be an ideal candidate as a multi-targeted 
A

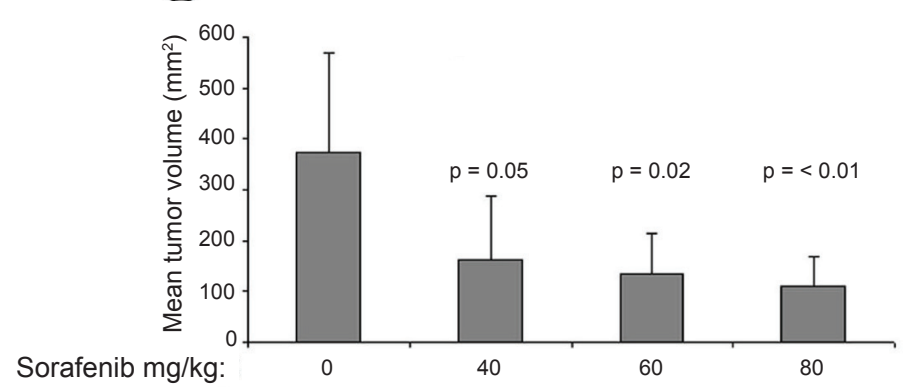

B
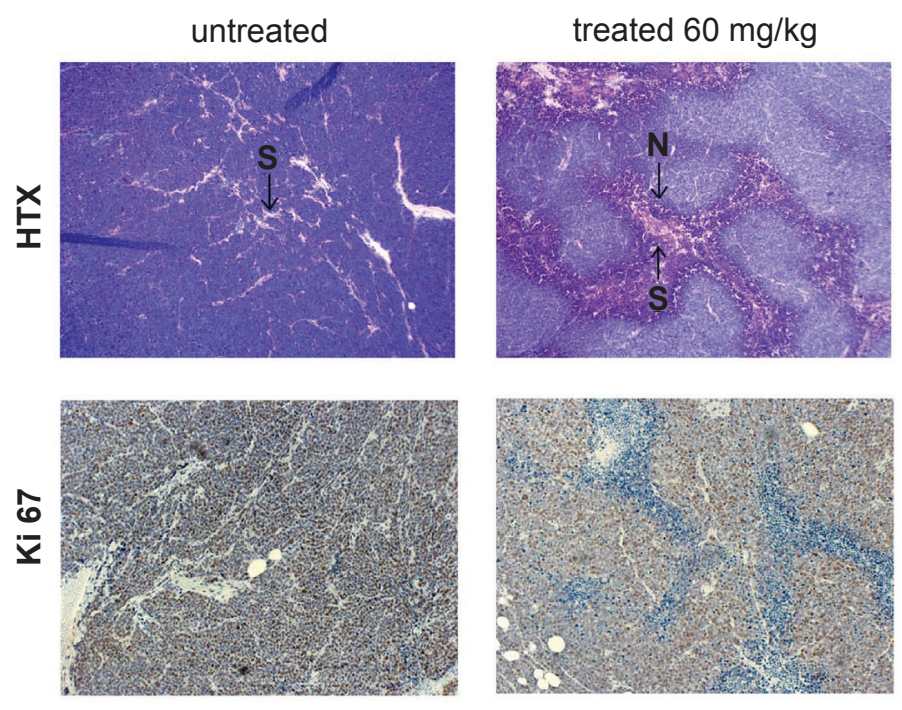

C
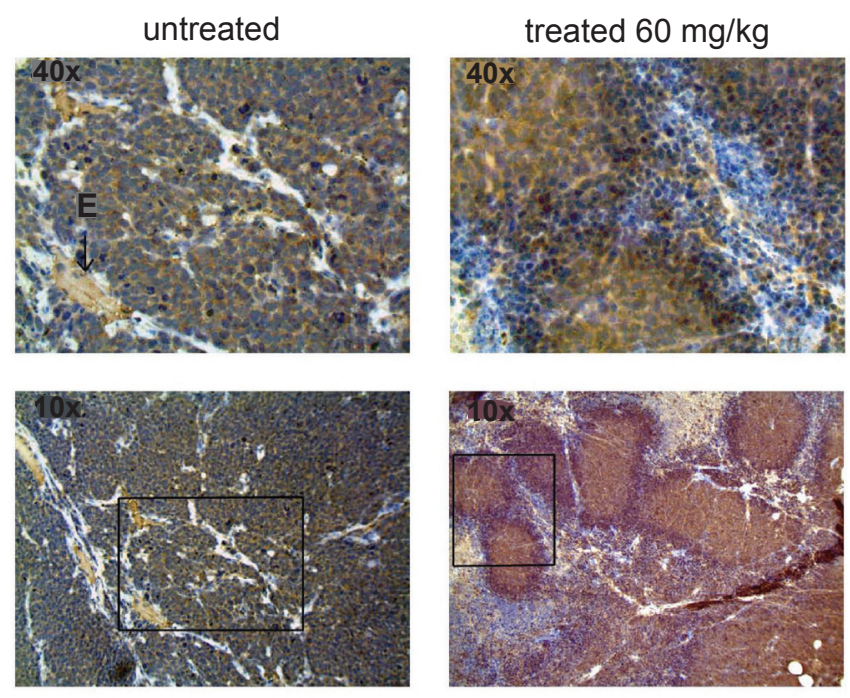

Figure 6 Effect of sorafenib on alveolar rhabdomyosarcoma (RMS) tumor xenografts. BALB/c SCID mice were inoculated subcutaneously with RMS cells. When the tumors were palpable, the mice were treated with $40,60,80 \mathrm{mg} / \mathrm{kg}$ body weight sorafenib or placebo per orally for 7 days. At the end point of the experiment, mice were euthanized, tumors were excised and fixed in formalin and stained immunohistochemically with HTX, Ki-67 and CD3I. A) Histogram showing the mean tumor mass at end point of experiment of rhabdomyosarcoma xenograft tumors treated with placebo or sorafenib. B) Hematoxilin (HX) and Ki-67 stained formalin-fixed paraffin embedded sections of RMS tumors. (N: necrosis, S: septa/fibrovascular tissue). C) Effect of sorafenib on tumor vascularization of formalin-fixed paraffin-embedded sections of RMS tumors were stained with $C D 31$.

Abbreviation: E, endothelial cells. 
therapy against the growth of several classes of soft tissue sarcoma. Cytotoxicity screening on a panel of soft tissue sarcoma cell lines of different histological subtypes (rhabdomyosarcomas, synovial sarcomas, Ewing's sarcomas and osteosarcomas) showed that sorafenib was indeed a potent inhibitor of cell proliferation. We found that alveolar rhabdomyosarcoma, synovial sarcoma and half of the Ewing's sarcoma cell lines tested displayed statistically significant inhibition of cell growth at clinically relevant concentrations $(<5 \mu \mathrm{M})$ in vitro.

Receptor tyrosine kinases generally promote cell growth by inducing phosphorylation and thus activation of substrate molecules, among which Erk, involved in promoting cell growth, and Akt, which can mediate cell survival, have been most studied. Since all three rhabdomyosarcoma cell lines responded to sorafenib, we analyzed the effect of sorafenib on inhibition of growth factor-mediated activation of Erk and Akt. In the case of rhabdomyosarcoma cells, sorafenib inhibited activation of both Erk and Akt in RMS cells in response to receptor tyrosine kinase activation by serum. Sorafenib also effectively blocked Erk activation in RH30 and RD cells; however, Akt was still activated in the presence of serum. The fact that Erk was inhibited suggests possible defects specific to the Akt pathway (eg, mutation) in these cell lines.

The fact that sorafenib failed to induce high levels of apoptosis in all cell lines tested, together with a reduced cell proliferation index in the tumor xenografts in the tumor cells, suggests in our case at least in part, that the anti-growth properties of sorafenib is due to blocking of cell proliferation. Since Erk is primarily a down stream effector for growth stimulatory signaling it would appear that inhibiting Erk activity more so than Akt is important for the anti tumor effect of sorafenib. In accordance with this a recent study has shown that IGF-1 stimulation of Erk phosphorylation in cholangiocarcinoma cells can be blocked by sorafenib (Huether et al 2007). In this study the authors point out two cell responses to sorafenib treatment, with cells either entering cycle arrest or undergoing apoptosis. Thus sorafenib would appear to have multiple outcomes depending on cell type.

Based on previous studies which show the over expression of IGF-1R in several rhabdomyosarcomas, we decided to include IGF-1R as a potential target for sorafenib. Indeed, we found IGF-1R to be highly over expressed in all 3 rhabdomyosarcoma cell lines tested in comparison with the other receptor tyrosine kinases known to be targeted by sorafenib (Figure 3). Using ligand specific activation assays, we showed that the IGF-1 ligand, as opposed to other growth factor ligands is a potent inducer of Erk and Akt activation, more importantly, however, this activation can be effectively blocked by pre treatment with sorafenib. Thus sorafenib widens its repertoire as a receptor tyrosine kinase antagonist by also inhibiting the IGF-1R pathways. Interestingly, our results seem to support the idea that IGF-1R is over expressed in these cell lines thus one could envision that rhabdomyosarcoma cell lines are hyper responsive to IGF-1 stimulation, thus by blocking the IGF-1 pathway a major growth stimulatory pathway is removed. Whether sorafenib inhibits IGF-1R directly by binding to the cell surface receptor or by inhibiting down stream components of the IGF-1R pathway such as c-Raf still remains to be determined. In the case of VEGFR, PDGFR, c-KIT and FLT-3 sorafenib has been shown to directly inhibit receptor autophosphorylation and activation upstream of Raf inhibition (Wilhelm et al 2006). In our case, sorafenib blocked IGF-1-mediated Akt activation in two of the three cell lines. Since the Erk and Akt pathways branch before c-Raf (a known sorafenib inhibitor) it would suggest direct interference with receptor activity in the sorafenib-treated cell lines which did not activate Akt. The relationship between IGF-1R auto-phosphorylation and sorafenib is an area of current research.

Building on this, rhabdomyosarcoma cells have been shown to have dependency on IGF signaling (El-Badry et al 1990; Minniti et al 1992; Minniti et al 1994; Shapiro et al 1994). Down regulation of IGF-1R expression by antisense has been shown to alter the malignant phenotype of rhabdomyosarcoma cells, resulting in tumor growth inhibition both in vitro and in vivo (Shapiro et al 1994). Moreover, the chimeric transcription factor, an exclusive feature of rhabdomyosarcoma, PAX3/7-FKHR has been shown to enhance direct transcriptional activator of the $I G F-1 R$ gene which may account for the relatively high levels of IGF-1R observed in these tumors (Gidding et al 2000; Barber et al 2002; Thimmaiah et al 2003).

Among the sarcoma cell lines, sorafenib had a growth inhibitory effect in soft tissue sarcomas that carry translocations but not in osteosarcomas, which typically display chaotic chromosome arrangements. The cell growth inhibition that we observed in the cell lines of soft tissue sarcomas was, however, not associated with the expression of cytogenetic markers.

Our in vivo study clearly showed a significant inhibition of rhabdomyosarcoma and synovial sarcoma (data not shown) growth. Xenografts of RMS cells gave rise to large, highly vascularized tumors characterized by high rates of 
proliferation. In contrast, in mice treated with sorafenib the tumors appeared smaller and were characterized by impaired tumor vascularization, large necrotic areas advanced to the tumor stroma and reduced proliferative capacity, supporting the notion that sorafenib can effectively block cell growth. These observations indicate that the activity of sorafenib on the fibrovascular tissue may deprive tumor cells of growth signaling and impairs vascularization, resulting in tumor cell death, and demonstrates that this drug affects both tumor cells and stromal cells (endothelial cells). The fact that sorafenibtreated tumors displayed poor vascularization also suggest that anti-angiogenic properties of this drug. We believe that in addition to the anti-proliferative effect of sorafenib on the rhabdomyosarcoma cells, its effect on the tumor stroma is determinant for the growth inhibition of the tumors.

Rhabdomyosarcoma is the most common soft tissue sarcoma in children (Bersani et al 2008). Current treatment for rhabdomyosarcoma is surgery, chemotherapy and radiotherapy. However, resistance to chemotherapy and radiation therapy is a limiting factor in the treatment of rhabdomyosarcoma, leading to therapeutic failure and limiting improved survival in these patients (Kuttesch 1996).

Up to date, the effect of sorafenib in vitro and in vivo have not been evaluated on rhabdomyosarcomas. Our results presented in this paper show that sorafenib can inhibit cell proliferation both in vitro and in vivo through the inhibition of IGF-1R-mediated signaling, and through impaired vascularization, indicating that patients with rhabdomyosarcoma that over express IGF-1R may be good candidates for treatment with sorafenib.

\section{Acknowledgments}

This work was supported by grants from The Cancer Society in Stockholm and the Swedish Children Cancer Foundation to BB. We are thankful to Dr Katia Scotlandi, Dr Janet Shipley and Dr Akira Kawai for providing sarcoma cell lines. We also thank Liss Garber, Margareta Rodensjö, Inger Bodin, Kenth Andersson and Margaretha Hagelin for technical assistance.

\section{References}

Adnane L, Trail PA, Taylor I, et al. 2006. Sorafenib (BAY 43-9006, Nexavar), a dual-action inhibitor that targets RAF/MEK/ERK pathway in tumor cells and tyrosine kinases VEGFR/PDGFR in tumor vasculature. Methods Enzymol, 407:597-612.

Ambrosini G, Cheema HS, Seelman S, et al. 2008. Sorafenib inhibits growth and mitogen-activated protein kinase signaling in malignant peripheral nerve sheath cells. Mol Cancer Ther, 4:890-6.

Amit I, Wides R, Yarden Y. 2007. Evolvable signaling networks of receptor tyrosine kinases: relevance of robustness to malignancy and to cancer therapy. Mol Syst Biol, 3:151.
Ammoun S, Flaiz C, Ristic N, et al. 2008. Dissecting and targeting the growth factor-dependent and growth factor-independent extracellular signal-regulated kinase pathway in human schwannoma. Cancer Res, 13:5236-45.

Armistead PM, Salganick J, Roh JS, et al. 2007. Expression of receptor tyrosine kinases and apoptotic molecules in rhabdomyosarcoma: correlation with overall survival in 105 patients. Cancer, 10:2293-303.

Ashcroft M, Ludwig RL, Woods DB, et al. 2002. Phosphorylation of HDM2 by Akt. Oncogene, 13:1955-62.

Barber TD, Barber MC, Tomescu O, et al. 2002. Identification of target genes regulated by PAX3 and PAX3-FKHR in embryogenesis and alveolar rhabdomyosarcoma. Genomics, 3:278-84.

Bersani F, Taulli R, Accornero P, et al. 2008. Bortezomib-mediated proteasome inhibition as a potential strategy for the treatment of rhabdomyosarcoma. Eur J Cancer, 6:876-84.

Bracarda S, Caserta C, Sordini L, et al. 2007. Protein kinase inhibitors in the treatment of renal cell carcinoma: sorafenib. Ann Oncol, 18:(Suppl 6):vi22-5.

Chambard JC, Lefloch L, Pouysségur J, et al. 2007. ERK implication in cell cycle regulation. Biochim Biophys Acta, 8:1299-310.

D’Adamo DR, Koeham ML, Schuetze S, et al. 2007. A multicenter phase II trial of oral sorafenib in non-GIST sarcomas CTEP trial \#7060. ASCO Annual Meeting, 10001.

Datta SR, Dudek H, Tao X, et al. 1997. Akt phosphorylation of BAD couples survival signals to the cell-intrinsic death machinery. Cell, 4:231-41.

del Peso L, González-García M, Page C, et al. 1997. Interleukin-3-induced phosphorylation of BAD through the protein kinase Akt. Science, 5338:687-9.

Do I, Araujo E, Kalil RK, et al. 2007. Protein expression of KIT and gene mutation of c-kit and PDGFRs in Ewing sarcomas. Pathol Res Pract, 3:127-34

Downward J. 1998. Mechanisms and consequences of activation of protein kinase B/Akt. Curr Opin Cell Biol, 2:262-7.

El-Badry OM, Minniti CP, Kohn EC, et al. 1990. Insulin-like growth factor II acts as an autocrine growth and motility factor in human rhabdomyosarcoma tumors. Cell Growth Differ, 1:325-31.

Escudier B, Eisen T, Stadler WM, et al. 2007. Sorafenib in advanced clear-cell renal-cell carcinoma. $N$ Engl J Med, 2:125-34.

Faivre S, Kroemer G, Raymond E. 2006. Current development of mTOR inhibitors as anticancer agents. Nat Rev Drug Discov, 8:671-88.

Flaherty KT. 2006. Chemotherapy and targeted therapy combinations in advanced melanoma. Clin Cancer Res, 7(Pt 2):2366s-70s.

Gee MF, Tsuchida R, Eichler-Jonsson C, et al. 2005. Vascular endothelial growth factor acts in an autocrine manner in rhabdomyosarcoma cell lines and can be inhibited with all-trans-retinoic acid. Oncogene, 54:8025-37.

Gidding CE, Germain GS, Dilling MB, et al. 2000. The influence of recombinant human insulin-like growth factor-I (rhIGF-I) on cell growth and cytotoxicity of drugs in childhood rhabdomyosarcoma cell lines and xenograft models. Cancer Chemother Pharmacol, 1:21-30.

Gordon T, McManus A, Anderson J, et al. 2001. Cytogenetic abnormalities in 42 rhabdomyosarcoma: a United Kingdom Cancer Cytogenetics Group Study. Med Pediatr Oncol, 2:259-67.

Handa A, Tokunaga T, Tsuchida T, et al. 2000 Neuropilin-2 expression affects the increased vascularization and is a prognostic factor in osteosarcoma. Int J Oncol, 2:291-5.

Huether A, Höpfner M, Baradari V, et al. 2007. Sorafenib alone or as combination therapy for growth control of cholangiocarcinoma. Biochem Pharmacol, 9:1308-17.

Karasarides M, Chiloeches A, Hayward R, et al. 2004. B-RAF is a therapeutic target in melanoma. Oncogene, 37:6292-8.

Kawai A, Naito N, Yoshida A, et al. 2004. Establishment and characterization of a biphasic synovial sarcoma cell line, SYO-1. Cancer Lett, $1: 105-13$.

Kreuter M, Paulssen M, Boeckeler J, et al. 2006. Clinical significance of vascular endothelial growth factor-A expression in Ewing's sarcoma. Eur J Cancer, 12:1904-11. 
Kuttesch JF JR, 1996. Multidrug resistance in pediatric oncology. Invest New Drugs, 1:55-67.

Landizzi L, de Giovanni C, Nicoletti G, et al. 2000. The metastatic ability of Ewing's sarcoma cells is modulated by stem cell factor and by its receptor c-kit. Am J Pathol, 6:2123-31.

Llovet J, Ricci S, Mazzaferro V, et al. 2007. Sorafenib improves survival in advanced hepatocellular carcinoma (HCC): Results of a Phase III randomized placebo-controlled trial (SHARP trial). ASCO Annual Meeting, LBA1.

Lowinger TB, Riedl B, Dumas J, et al. 2002. Design and discovery of small molecules targeting raf-1 kinase. Curr Pharm Des, 8:2269-78.

Maki RG, Koehan ML, Undevia SD, et al. 2008. Updated results of a phase II study of oral multi-kinase inhibitor sorafenib in sarcomas, CTEP study \#7060. ASCO Annual Meeting, 10531.

Merighi S, Benini A, Mirandola P, et al. 2006. Modulation of the Akt/ Ras/Raf/MEK/ERK pathway by A(3) adenosine receptor. Purinergic Signal, 4:627-32.

Minniti CP, Kohn EC, Grubb JH, et al. 1992. The insulin-like growth factor II (IGF-II)/mannose 6-phosphate receptor mediates IGF-II-induced motility in human rhabdomyosarcoma cells. $J$ Biol Chem, 13:9000-4.

Minniti CP, Tsokos M, Newton WA JR, et al. 1994. Specific expression of insulin-like growth factor-II in rhabdomyosarcoma tumor cells. $\mathrm{Am} \mathrm{J}$ Clin Oncol, 2:198-203.

Misra UK, Pizzo SV. 2004. Potentiation of signal transduction mitogenesis and cellular proliferation upon binding of receptor-recognized forms of alpha2-macroglobulin to 1-LN prostate cancer cells. Cell Signal, 4:487-96.

Myhre O, Sterri SH, Bogen IL, et al. 2004. Erk1/2 phosphorylation and reactive oxygen species formation via nitric oxide and Akt-1/Raf-1 crosstalk in cultured rat cerebellar granule cells exposed to the organic solvent 1,2,4-trimethylcyclohexane Toxicol Sci, 2:296-303.

Panka DJ, Wang W, Atkins MB, et al. 2006. The Raf inhibitor BAY 43-9006 (Sorafenib) induces caspase-independent apoptosis in melanoma cells. Cancer Res, 3:1611-9.

Ratain MJ, Eisen T, Stadler WM, et al. 2006. Phase II placebo-controlled randomized discontinuation trial of sorafenib in patients with metastatic renal cell carcinoma. J Clin Oncol, 16:2505-12.

Ricotti E, Fagioli F, Garelli E, et al. 1998. c-kit is expressed in soft tissue sarcoma of neuroectodermic origin and its ligand prevents apoptosis of neoplastic cells. Blood, 7:2397-405.

Ryan CW, von Mwhren M, Rankin CJ, et al. 2008. Phase II intergroup study of sorafenib (S) in advanced soft tissue sarcomas (STS): SWOG 0505. ASCO Annual Meeting, 10532.
Shapiro DN, Jones BG, Shapiro LH, et al. 1994. Antisense-mediated reduction in insulin-like growth factor-1 receptor expression suppresses the malignant phenotype of a human alveolar rhabdomyosarcoma. $J$ Clin Invest, 3:1235-42.

Sridhar SS, Hedley D, Siu LL, 2005. Raf kinase as a target for anticancer therapeutics. Mol Cancer Ther, 4:677-85.

Tamborini E, Bonadiman L, Greco A, et al. 2004. Expression of ligand-activated KIT and platelet-derived growth factor receptor beta tyrosine kinase receptors in synovial sarcoma. Clin Cancer Res, 3:938-43.

Thimmaiah KN, Easton J, Huang S, et al. 2003. Insulin-like growth factor I-mediated protection from rapamycin-induced apoptosis is independent of Ras-Erk1-Erk2 and phosphatidylinositol 3'-kinase-Akt signaling pathways. Cancer Res, 2:364-74.

Tong FK, Chow S, Hedley D, 2006. Pharmacodynamic monitoring of BAY 43-9006 (Sorafenib) in phase I clinical trials involving solid tumor and AML/MDS patients, using flow cytometry to monitor activation of the ERK pathway in peripheral blood cells. Cytometry. Part B, Clin Cytometry, 3:107-14.

Wiebe L, Kasza KE, Maki RG, et al. 2008. Activity of sorafenib (SOR) in patients (pts) with imatinib (IM) and sunitinib (SU)-resistant (RES) gastrointestinal stromal tumors (GIST): A phase II trial of the University of Chicago Phase II Consortium. ASCO Annual Meeting, 10502.

Wilhelm S, Carter C, Lynch M, et al. 2006. Discovery and development of sorafenib: a multikinase inhibitor for treating cancer. Nat Rev Drug Discov, 10:835-44.

Wilhelm SM, Carter C, Tang L, et al. 2004. BAY 43-9006 exhibits broad spectrum oral antitumor activity and targets the RAF/MEK/ERK pathway and receptor tyrosine kinases involved in tumor progression and angiogenesis. Cancer Res, 19:7099-109.

Xue L, Murray JH, Tolkovsky AM, 2000. The Ras/phosphatidylinositol 3-kinase and Ras/ERK pathways function as independent survival modules each of which inhibits a distinct apoptotic signaling pathway in sympathetic neurons. $J$ Biol Chem, 12:8817-24.

Yu C, Friday BB, Lai JP, et al. 2006. Cytotoxic synergy between the multikinase inhibitor sorafenib and the proteasome inhibitor bortezomib in vitro: induction of apoptosis through Akt and c-Jun NH2-terminal kinase pathways. Mol Cancer Ther, 9:2378-87.

Zhang L, Yu D, Hu M, et al. 2000. Wild-type p53 suppresses angiogenesis in human leiomyosarcoma and synovial sarcoma by transcriptional suppression of vascular endothelial growth factor expression. Cancer Res, 133655-61.

Zhong H, Bowen J. 2007. Molecular design and clinical development of VEGFR kinase inhibitors. Curr Topic Med Chem, 7:1379-93. 